\title{
Channel Holding Time of Packet Sessions in All-IP Cellular Networks
}

\author{
Jeng-Feng Weng*, Bying-He Ku ${ }^{\dagger}$, Jyh-Cheng Chen*and Wen-Tsuen Chen ${ }^{\dagger}$ \\ ${ }^{*}$ Department of Computer Science, National Chiao Tung University, Hsinchu, Taiwan. \\ ${ }^{\dagger}$ Institute of Information Science, Academia Sinica, Taiwan.
}

\begin{abstract}
In this paper, we analyze the packet session channel holding time for packet-switched cellular networks. Channel holding time depends on mobility which is characterized by cell residence time in this paper. We apply HyperErlang distribution model to investigate the packet session channel holding time in which a session will experience active and idle periods. In terms of new session, handoff session in busy mode, handoff session with new arrival packets in idle mode and handoff session without arrival packet in idle mode, we demonstrate that the effective channel holding time is exponentially distributed if and only if the cell residence time is exponentially distributed. The analytical results provide a new approach to evaluate traffic performance and system design in packet-switched cellular networks.
\end{abstract}

Keywords-channel holding time, packet session, cell residence time, idle mode, mobility, analysis

\section{INTRODUCTION}

In cellular networks, channel holding time, which depends on user's mobility, is an important quantify for network design and performance analysis [1]. In traditional CircuitSwitched (CS) cellular networks, voice traffic is transmitted in dedicated channel in which the resource is reserved for the whole duration of the call. In [2]-[4], Fang et al. propose a new mobility mode, called the Hyper-Erlang distribution model, to analyze the channel holding time in CS cellular networks. For an exponential call holding time and Poisson new call arrivals, they show that the channel holding time is exponentially distributed if and only if the cell residence time is also exponentially distributed. Also, the new call channel holding time and the handoff call channel holding time have the same distribution if and only if the cell residence time is exponentially distributed.

In Packet-Switched (PS) cellular networks, however, the behavior of a packet session is different with that of a voice call. The major difference is that during a packet session, sometimes a User Equipment (UE) may sleep. Therefore, the resource is not reserved for the whole duration of a packet session. For example, LTE/LTE-A defines Discontinuous Reception (DRX) [5] to save energy. With DRX, an UE turns on the receiver at some pre-defined time points while sleeps at other time periods. In this paper, we propose a new model to analyze channel holding time for packet sessions. Same as that in [2]-[4], [6]-[8], channel holding time depends on user's mobility which is characterized by cell residence time. We apply Hyper-Erlang distribution model to investigate the packet session channel holding time

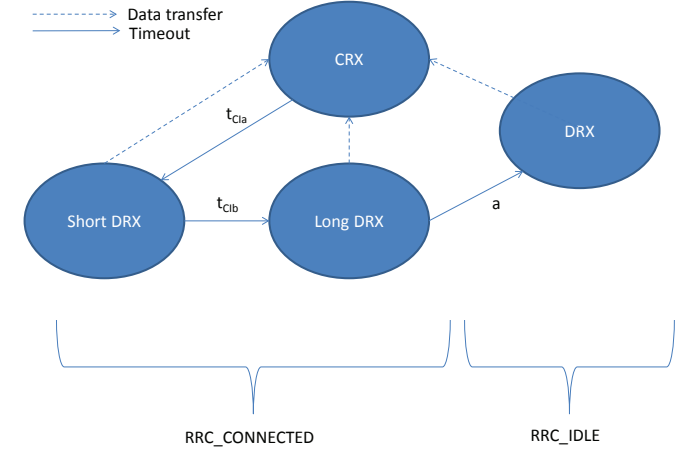

Figure 1. Discontinuous reception (DRX) in LTE

in which a session will experience active and idle periods. Similar to CS cellular networks, we demonstrate: (1) Packet session channel holding time is exponentially distributed if and only if the cell residence time is exponentially distributed, and (2) new packet session channel holding time and handoff packet session channel holding time have the same distribution if and only if the cell residence time is exponentially distributed. Although we use LTE DRX as an example in this paper, the model we develop can be used in other PS networks which have similar behavior. The analytical model presented in this paper can be used as a guideline for network design and performance analysis.

\section{Discontinuous Reception (DRX) Mode In LTE}

Because the demand of high bandwidth for data transfer may drain the battery power of an UE quickly, LTE/LTE-A defines Discontinuous Reception (DRX) [5] to save energy. With DRX, an UE turns on the receiver at some pre-defined time points while sleeps at other time periods. It is an important issue how to select proper DRX parameters, not only to increase UE's battery lifetime, but also to better utilize resource and minimize packet delay. The DRX state machine in LTE is shown in Fig. 1, in which UE can be in two different states: RRC_CONNECTED and RRC_IDLE to represent whether the Radio Resource Control (RRC) is connected or idle. In RRC_CONNECTED state, the radio moves from Continuous Reception (CRX) to Short DRX state, and then Long DRX state when it is waiting for data. If the channel is idle longer than $a$, the RRC then will move from RRC_CONNECTED to RRC_IDLE. In this paper, we consider the channel holding time when an UE 

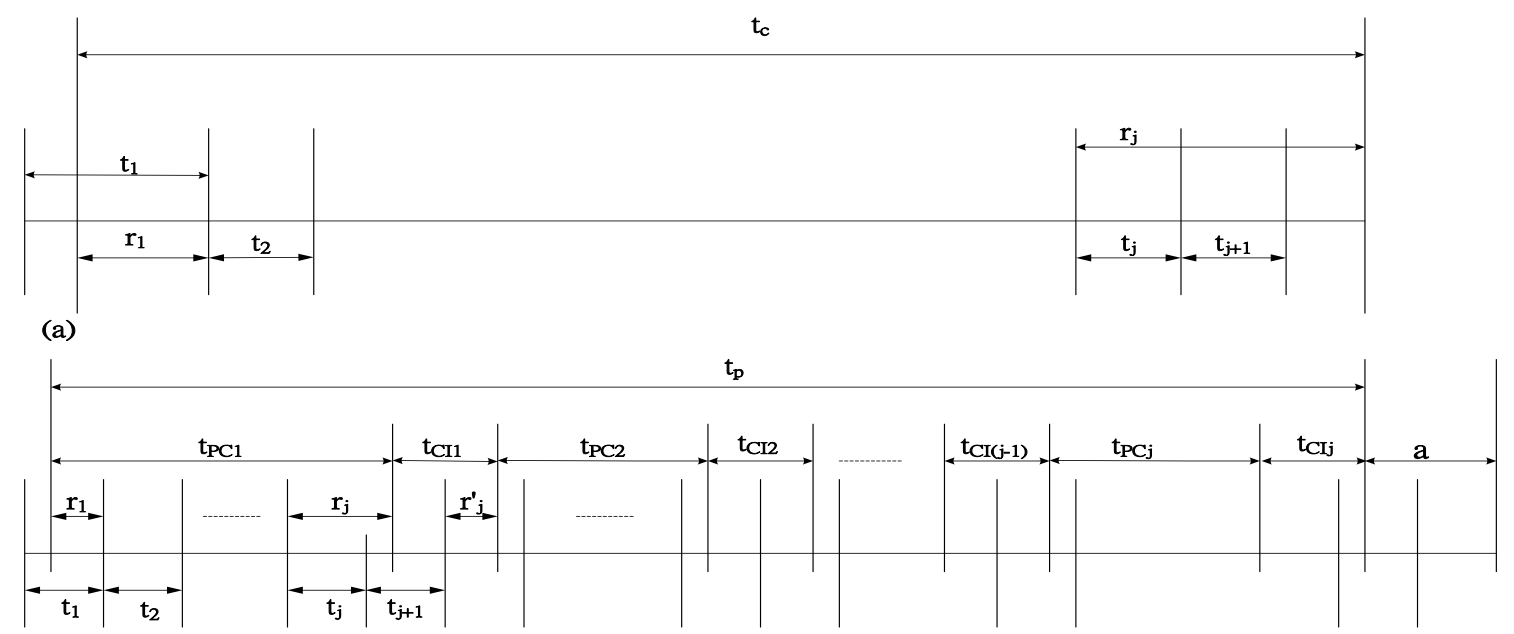

(b)

Figure 2. (a) Timing diagram for CS cellular networks, (b) Timing diagram for PS cellular networks

is in RRC_CONNECTED state. As shown in Fig. 1, even in RRC_CONNECTED, an UE may still sleep in long/short DRX state.

\section{Analytical Model of Packet Session Channel Holding Time}

Fig. 2 (a) shows the timing diagram for the call holding time and cell residence time in CS cellular networks [2][4]. In Fig. 2 (a), $t_{c}$ is the duration of a channel holding time. As that in [2]-[4], $t_{c}$ is exponentially distribution with parameter $\mu$. Let $t_{n h}$ and $t_{h h}$ denote the channel holding times for a new call and a handoff call, respectively. Fang et al. propose the channel holding for a new call as:

$$
t_{n h}=\min \left\{t_{c}, r_{1}\right\}
$$

The channel holding time for a handoff call is:

$$
t_{h h}=\min \left\{r_{j}, t_{j}\right\}
$$

Next, we present our analytical model for $t_{p}$, the packet session channel holding time which is the time an UE in the RRC_CONNECTED state as shown in Fig. 1. As discussed in Section II, even in RRC_CONNECTED state, an UE may still sleep in long/short DRX state. Fig. 2 (b) illustrates the DRX cycles of length $t_{p}=\sum t_{P C j}+\sum t_{C I j}$, where $j=$ $1,2, \ldots$, for channel holding time and cell residence time of a packet session. During $t_{P C 1}$, the channel is used for packet transmission. If there is no packet, the UE enters sleep mode, which is denoted as $t_{C I 1}$. Once there is a packet arrival, the $\mathrm{UE}$ changes to $t_{P C 2}$. The $t_{P C j}$ essentially is corresponding to the CRX state in Fig. 1 . The $t_{C I j}$ includes the Short DRX and Long DRX in Fig. 1, in which the channel is idle. The maximum idle time is denoted as $a$. If the channel is idle longer than $a$, the UE enters RRC_IDLE and we consider the packet session is finished.
Let $t_{C I j}$ be the Connected-Idle time that the UE has no activity in both downlink (forward link) and uplink (reverse link). The maximum value of $t_{C I j}$ is the length of the idle timer $a$. Let $r_{1}$ be the period from the time a new packet session starts to the time the session leaves the serving cell but this new session is not finished yet. Let $t_{j}$ be the cell residence time (or dwell time) and $r_{j}(j \geq 2)$ be the residual life time distribution of the packet session holding time while the packet session completes $j^{\text {th }}$ handoff successfully. Table I list the parameters used in this paper.

In PS networks, let $t_{e}$ represent the effective packet session holding time. We then can obtain:

$$
t_{e}=t_{p}+a, t_{C I j}>a
$$

From Fig. 2(b), we have the following four effective channel holding time.

1) $t_{p n h}$ : the effective channel holding time for a new session [3], [9] is:

$$
t_{p n h}=\min \left\{t_{p}+a, r_{1}\right\}
$$

We assume that $\lambda$ is the new session arrival rate to a cell.

2) $t_{\text {ph__busy }}$ : the effective channel holding time for a handoff session in busy model [3], [9] is:

$$
t_{\text {phh_busy }}=\min \left\{r_{j}+a, t_{j}\right\}
$$

We assume that $\lambda_{h b}$ is the arrival rate to a cell with handoff session in busy model.

3) $t_{\text {phh_idle_new }}$ : the effective channel holding time for a handoff session with new arrival packets in idle model is:

$$
t_{p h h_{-} i d l e \_n e w}=\min \left\{r_{j}+a, t_{j}\right\}
$$

We assume that $\lambda_{h d a}$ is the arrival rate to a cell with handoff session with new arrival packets in idle model. 
Table I

LIST OF PARAMETERS

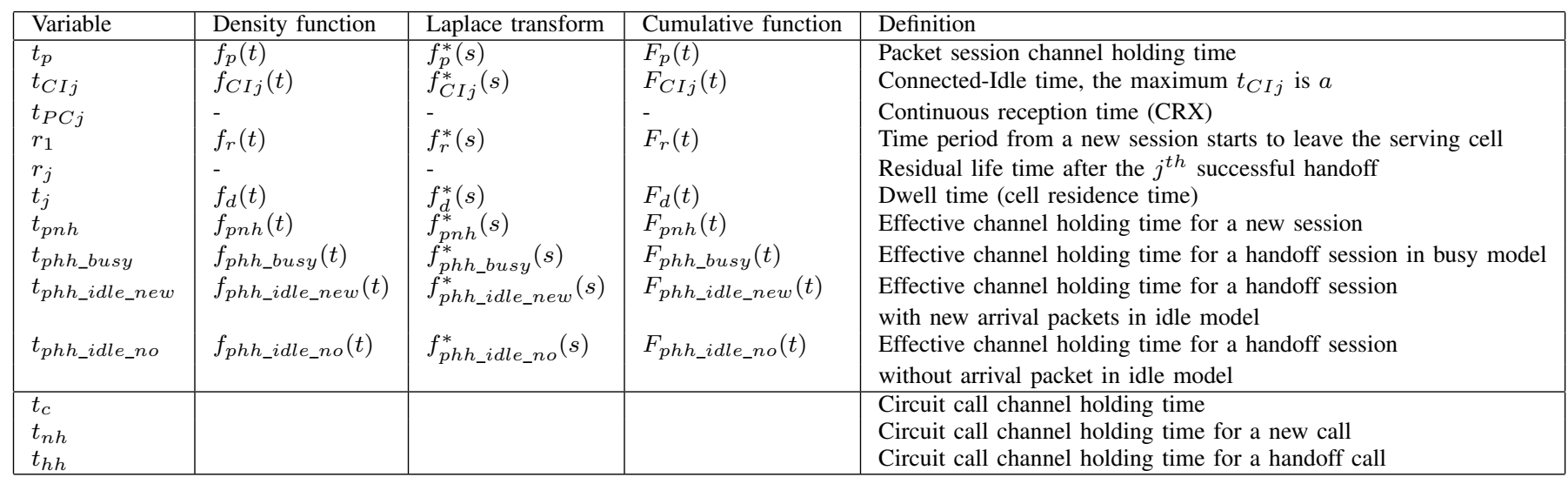

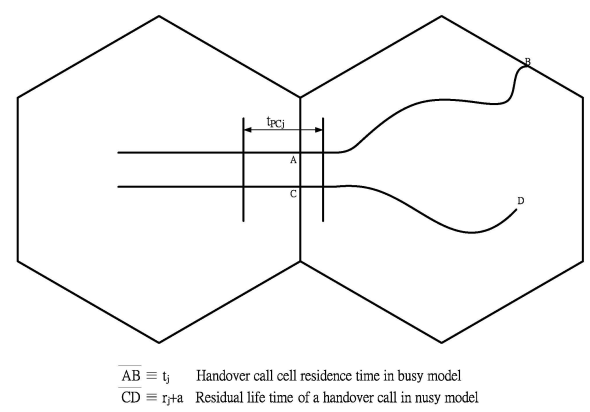

Figure 3. Cell residence time and residual life time of a handover session

4) $t_{\text {phh_idle_no: }}$ the effective channel holding time for a handoff session with no arrival packets in idle model is:

$$
t_{p h h_{-} i d l e_{-} n o}=\min \left\{r_{j}^{\prime}+a, t_{j}\right\}
$$

We assume that $\lambda_{h d b}$ is the arrival rate to a cell with handoff session without arrival packet in idle model.

Noted that in Fig. 3, when a user moves from the left cell to the right cell in busy model, the user will be in idle mode when the user completes the data transmission session in the right cell. On the other hand, in Fig. 4, when a user moves from the left cell to the right cell in idle model, $T_{C I j}<$ $a$ and a data transmit requirement are needed in the right cell. In this case, the cell residence time $T_{j}$ is part of $T_{C I j}$. Because $r_{j}$ is the residual lift time after the $j$-th successful handoff and the user will finally be in long DRX mode (i.e., the maximum value of $t_{C I j}=a$ ), we have (5) $\equiv(6)$.

Next, let the density functions of $t_{p}, t_{C I j}, t_{j}, r_{1}$, $t_{\text {pnh }}, t_{\text {phh_busy }}, t_{\text {phh_idle_new }}$ and $t_{\text {phh_idle_no }}$ be $f_{p}(t)$, $f_{C I j}(t), f_{d}(t), f_{r}(t), f_{\text {pnh }}(t), f_{\text {phh_busy }}(t), f_{\text {phh_idle_new }}(t)$ and $f_{p h h_{-} i d l e_{-} n o}(t)$, respectively. The corresponding Laplace transforms of these density functions are $f_{p}^{*}(s), f_{C I j}^{*}(s)$,

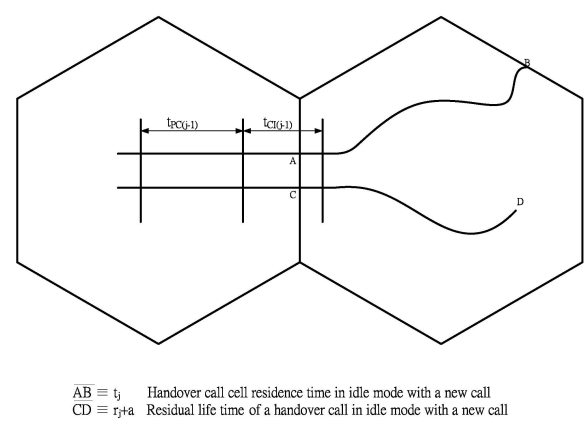

Figure 4. Cell residence time and residual life time of a handover session in idle mode with a new session

$f_{d}^{*}(s), f_{r}^{*}(s), f_{\text {pnh }}^{*}(s), f_{\text {phh_busy }}^{*}(s), f_{\text {phh_idle_new }}^{*}(s)$ and $f_{\text {phh_idle_no }}^{*}(s)$, respectively. Their cumulative distribution functions are $F_{p}(t), F_{C I j}(t), F_{d}(t), F_{r}(t), F_{p n h}(t)$, $F_{p h h_{-} b u s y}(t), F_{p h h_{-} i d l e \_n e w}(t)$ and $F_{p h h_{-} i d l e \_n o}(t)$, respectively.

\section{A. Handoff Session in Busy Model}

In this section, we will show that the effective channel holding time for a handoff session in busy model $\left(t_{\text {phh_busy }}\right)$ is exponential distribution if and only if the cell residence time $\left(t_{j}\right)$ is exponentially distributed. First, We can obtain the conditional probability of (5):

$$
\begin{aligned}
& P\left(t_{p h h_{\text {b busy }}} \leq t \mid t_{C I j}>a\right) \\
& =P\left(t_{p} \leq t-a\right)+P\left(t_{j} \leq t\right)-P\left(t_{p} \leq t-a\right) P\left(t_{j} \leq t\right)
\end{aligned}
$$

where we have $P\left(\left(r_{j}+a\right) \leq t, t_{j} \leq t\right)=P\left(\left(r_{j}+\right.\right.$ $a) \leq t) P\left(t_{j} \leq t\right)$ from the independence of $r_{j}, t_{j}$ and $t_{C I j}$ [2], [3], [9]. From the Residual Life Theorem [10] or the argument in [11], we also have $P\left(\left(r_{j}+a\right) \leq\right.$ $t)=P\left(t_{p} \leq t-a\right)$ from the memoryless property of the 
exponential distribution, where $r_{j}$ has the same distribution as $t_{p}$.

By differentiating (8), we have the conditional density:

$$
\begin{aligned}
& \quad f_{\text {ph__busy }}(t)=f_{p}(t-a)+f_{d}(t) \\
& \quad-f_{p}(t-a) P\left(t_{j} \leq t\right)-P\left(t_{p} \leq t-a\right) f_{d}(t) \\
& =f_{p}(t-a)-f_{p}(t-a) \cdot \int_{0}^{t} f_{d}(\tau) d \tau \\
& \quad+f_{d}(t)-f_{d}(t) \cdot \int_{0}^{t} f_{p}(\tau-a) d \tau
\end{aligned}
$$

Suppose that the cell residence times $t_{j}$ is exponentially distributed with parameter $\eta$, then, from (9), we obtain:

$$
\begin{aligned}
f_{\text {phh_busy }}(t) & =\mu e^{-\mu(t-a)} \times e^{-\eta t}+\eta e^{\eta t} \times e^{-\mu(t-a)} \\
& =(\mu+\eta) e^{-(\mu+\eta)\left(t-\frac{\mu a}{\mu+\eta}\right)}
\end{aligned}
$$

which is a Two-Parameter Exponential Distribution [12]. Contrariwise, suppose that the effective channel holding time for a handoff session in busy model is exponential distribution with parameter $\gamma$. Let $Z(t)=\int_{t}^{\infty} f_{d}(\tau) d \tau$, then $Z(t)^{\prime}=-f_{d}(t)$. From (9), we get:

$$
\mu e^{-\mu(t-a)} Z(t)+e^{-\mu(t-a)} f_{d}(t)=\gamma e^{-\gamma \tau}
$$

From (11), we can derive:

$$
\begin{aligned}
Z(t) & =e^{\mu(t-a)} Z(0)+\int_{0}^{t} e^{\mu[(t-a)-\tau]}\left[-\gamma e^{-(\gamma-\mu) \tau}\right] d \tau \\
& =e^{\mu a} \cdot e^{-(\gamma-\mu) t}
\end{aligned}
$$

Thus, $Z(t)^{\prime}=-(\gamma-\mu) e^{[-(\gamma-\mu) t+\mu a]}$. Finally, we obtain:

$$
\begin{array}{r}
f(t)=(\gamma-\mu) e^{[-(\gamma-\mu) t+\mu a]} \\
=(\gamma-\mu) e^{-(\gamma-\mu)\left(t-\frac{\mu a}{\gamma-\mu}\right)} .
\end{array}
$$

From (13), we prove that if the effective channel holding time for a handoff session in busy model is exponential distribution, the cell residence time must be exponentially distributed.

\section{B. New Session}

Similarly, the conditional probability of (4) is:

$$
\begin{aligned}
& P\left(t_{p n h} \leq t \mid t_{C I j}>a\right) \\
& =P\left(t_{p} \leq t-a\right)+P\left(r_{1} \leq t\right)-P\left(t_{p} \leq t-a\right) P\left(r_{1} \leq t\right) .
\end{aligned}
$$

By differentiating above equation, we obtain:

$$
\begin{aligned}
f_{p n h}(t) & =f_{p}(t-a)+f_{r}(t) \\
- & f_{p}(t-a) P\left(r_{1} \leq t\right)-P\left(t_{p} \leq t-a\right) f_{r}(t) \\
& =f_{p}(t-a)-f_{p}(t-a) \cdot \int_{0}^{t} f_{r}(\tau) d \tau \\
+ & f_{r}(t)-f_{r}(t) \cdot \int_{0}^{t} f_{p}(\tau-a) d \tau .
\end{aligned}
$$

Suppose that the effective channel holding time of a new session is exponentially distributed with parameter $\mu_{1}$. From a similar derivation of the effective channel holding time for a handoff session in busy model which is exponential distribution, we can deduce that:

$$
f_{r}(t)=\left(\mu_{1}-\mu\right) e^{-\left(\mu_{1}-\mu\right)\left(t-\frac{\mu a}{\mu_{1}-\mu}\right)}
$$

which is also an exponential distribution.

\section{Handoff Session in Idle with New Arrival Packets}

The effective channel holding time for a handoff session with new arrival packets in idle model in (6) is the same as that for a handoff session in busy model in (5). Based on III-A, we can derive that the effective channel holding time for a handoff session with new arrival packets in idle model is exponential distribution if and only if the cell residence time is exponential distribution.

\section{Handoff Session in Idle without Arrival Packet}

From (7), we get the conditional probability:

$$
\begin{gathered}
P\left(t_{p h h \_i d l e \_n o} \leq t \mid t_{C I j}>a\right) \\
=P\left(t_{p} \leq t-a\right)+P\left(t_{j} \leq t\right) \\
-P\left(t_{p} \leq t-a\right) P\left(t_{j} \leq t\right)
\end{gathered}
$$

where we have the independence of $t_{C I j}$ and $t_{j}$ [3], [9], and the independence of $t_{j}$ and $r_{j}^{\prime}$ [3], [9]. We also apply $P\left(\left(r_{j}^{\prime}+a\right) \leq t\right)=P\left(t_{p} \leq t-a\right)$ from the memoryless property of the exponential distribution. Thus, $r_{j}^{\prime}$ has the same distribution as $t_{p}$. Therefore, we have the same conditional density and the Laplace transform with (8) and (19), respectively.

Theorem 1: For a PS network with exponential session holding time and Poisson new session arrivals, we can state:

1) The effective channel holding time for a new session is exponentially distributed if and only if the cell residence time is exponentially distributed.

2) The effective channel holding time for a handoff session in busy model is exponentially distributed if and only if the cell residence time is exponentially distributed.

3) The effective channel holding time for a handoff session in idle model with new or no arrival packets is exponentially distributed if and only if the cell residence time is exponentially distributed.

4) The effective channel holding time is exponentially distributed if and only if the cell residence time is exponentially distributed.

Let $t_{p}$ denote the packet session channel holding time (i. e., the channel holding time no matter whether the session is new or handoff session). Thus $t_{p}=t_{p n h}$ with probability $\lambda /\left(\lambda+\lambda_{h b}+\lambda_{h d a}+\lambda_{h d b}\right), t_{p}=t_{p h h_{-} b u s y}$ with probability $\lambda_{h b} /\left(\lambda+\lambda_{h b}+\lambda_{h d a}+\lambda_{h d b}\right)$ and so on. We obtain that: 


$$
\begin{aligned}
& f_{p}^{*}(s)=\frac{\lambda}{\lambda+\lambda_{h b}+\lambda_{h d a}+\lambda_{h d b}} f_{p n h}^{*}(s) \\
& \quad+\frac{\lambda_{h b}}{\lambda+\lambda_{h b}+\lambda_{h d a}+\lambda_{h d b}} f_{p h h-b u s y}^{*}(s) \\
& \quad+\frac{\lambda_{h d a}}{\lambda+\lambda_{h b}+\lambda_{h d a}+\lambda_{h d b}} f_{p h h-i d l e-n e w}^{*}(s) \\
& \quad+\frac{\lambda_{h d b}}{\lambda+\lambda_{h b}+\lambda_{h d a}+\lambda_{h d b}} f_{p h h-i d l e-n o}^{*}(s)
\end{aligned}
$$

We assume that $\lambda_{h d a}=\lambda_{h b}$ and $\lambda_{h d b}=0$. From (18) we have:

$$
\begin{aligned}
& f_{p}^{*}(s)=\frac{\lambda}{2 \lambda+\lambda_{h b}}\left(f_{p n h}^{*}(s)+f_{p h h \_i d l e \_n e w}^{*}(s)\right) \\
& +\frac{\lambda_{h b}}{2 \lambda+\lambda_{h b}} f_{p h h_{-} \text {busy }}^{*}(s) .
\end{aligned}
$$

Applying Laplace transform to (9), we obtain:

$$
\begin{aligned}
& f_{\text {phh_busy }}^{*}(s) \\
& =f_{d}^{*}(s)+e^{-a s} f_{p}^{*}(s)-\int_{0}^{\infty} e^{-s t}\left[f_{d}(t) \int_{0}^{t} f_{p}(\tau-a) d \tau\right] d t \\
& \quad-\int_{0}^{\infty} e^{-s t}\left[f_{p}(t-a) \int_{0}^{t} f_{d}(\tau) d \tau\right] d t \\
& =e^{-a s} \frac{\mu}{s+\mu}+e^{\mu a}\left(\frac{s}{s+\mu}\right) f_{d}^{*}(s+\mu)
\end{aligned}
$$

where we apply the theorem that $\mathcal{L}\left(g^{\prime}(t)\right)=s \mathcal{L}(g(t))-g(0)$ if $g$ is continuous at $t=0$. Here, $g(t)=F_{d}(t), g^{\prime}(t)=f_{d}(t)$ and $f_{d}(0)=0$.

\section{E. New Session}

Thus, the Laplace transform of (15) is:

$$
\begin{aligned}
& f_{p n h}^{*}(s) \\
& =f_{r}^{*}(s)+e^{-a s} f_{p}^{*}(s)-\int_{0}^{\infty} e^{-s t}\left[f_{r}(t) \int_{0}^{t} f_{p}(\tau-a) d \tau\right] d t \\
& \quad-\int_{0}^{\infty} e^{-s t}\left[f_{p}(t-a) \int_{0}^{t} f_{r}(\tau) d \tau\right] d t \\
& =e^{-a s} \frac{\mu}{s+\mu}+e^{\mu a}\left(\frac{s}{s+\mu}\right) f_{r}^{*}(s+\mu) .
\end{aligned}
$$

If the residual life time $r_{1}$ of $t_{1}$ is exponentially distributed with parameter $\mu_{r}$, the Laplace transform of $r_{1}\left(f_{r}^{*}(s)\right)$ is $\mu_{r} /\left(s+\mu_{r}\right)$. Taking this into (20), we obtain:

$$
\begin{aligned}
f_{p n h}^{*}(s) & =e^{-a s} \frac{\mu}{s+\mu}+e^{\mu a}\left(\frac{s}{s+\mu}\right) f_{r}^{*}(s+\mu) \\
= & e^{-a s} \frac{\mu}{s+\mu}+e^{\mu a} \frac{\mu_{r} s}{(s+\mu)\left(s+\mu+\mu_{r}\right)} .
\end{aligned}
$$

Applying simple poles (see Appendix) or looking up (20) in the table of inverse Laplace transforms, we can obtain:

$$
\begin{aligned}
& f_{p n h}(t) \\
& =u_{a}(t) \mu e^{-\mu(t-a)}+e^{\mu a} \frac{\mu_{r}\left(-\mu e^{-\mu t}-\left(-\mu-\mu_{r}\right) e^{-\left(\mu+\mu_{r}\right) t}\right)}{-\mu-\left(-\mu-\mu_{r}\right)} \\
& =u_{a}(t) \mu e^{-\mu(t-a)}-e^{\mu a} \mu e^{-\mu t}+e^{\mu a}\left(\mu+\mu_{r}\right) e^{-\left(\mu+\mu_{r}\right) t} . \text { (22) }
\end{aligned}
$$

Similarly, if $t_{j}$ is exponentially distributed with parameter $\mu_{d}$,

$$
\begin{aligned}
& f_{p h h}(t) \\
& =u_{a}(t) \mu e^{-\mu(t-a)}+e^{\mu a} \frac{\mu_{d}\left(-\mu e^{-\mu t}-\left(-\mu-\mu_{d}\right) e^{-\left(\mu+\mu_{d}\right) t}\right)}{-\mu-\left(-\mu-\mu_{d}\right)} \\
& =u_{a}(t) \mu e^{-\mu(t-a)}-e^{\mu a} \mu e^{-\mu t}+e^{\mu a}\left(\mu+\mu_{d}\right) e^{-\left(\mu+\mu_{d}\right) t} .(23)
\end{aligned}
$$

\section{F. Handoff Session in Idle with New Arrival Packets}

The effective channel holding time for a handoff session with new arrival packets in idle model in (6) is the same as that for a handoff session in busy model in (5). We have the same conditional density and the Laplace transform with (8) and (19), respectively.

\section{G. Handoff Session in Idle without Arrival Packet}

Because the conditional density function of the handoff session in idle without arrival packet in (17) is the same as the conditional density function of the handoff session in busy in (8), the Laplace transform of (17) is also the same as (19).

Theorem 2: For a PS network with exponential session holding time and Poisson new session arrival rate $\lambda$, we have the following statements:

1) The Laplace transform of the probability density function of the effective channel holding time for a new session is given by:

$$
f_{p n h}^{*}(s)=e^{-a s} \frac{\mu}{s+\mu}+e^{\mu a}\left(\frac{s}{s+\mu}\right) f_{r}^{*}(s+\mu) .
$$

2) The Laplace transform of the probability density function of the effective channel holding time for a handoff session in busy model or a handoff session with new arrival packets or a handoff session without arrival packet in idle model is given by:

$$
\begin{aligned}
& f_{\text {phh_busy }}^{*}(s)=f_{\text {phh_idle_no }}^{*}(s)=f_{\text {phh_idle_new }}^{*}(s) \\
& =e^{-a s} \frac{\mu}{s+\mu}+e^{\mu a}\left(\frac{s}{s+\mu}\right) f_{d}^{*}(s+\mu) .
\end{aligned}
$$

3) The Laplace transform of the probability density function of the effective channel holding time is given by:

$$
\begin{aligned}
& f_{p}^{*}(s)=\frac{\lambda}{2 \lambda+\lambda_{h b}}\left(f_{p n h}^{*}(s)+f_{p h L_{-} \text {idle_new }}^{*}(s)\right) \\
& +\frac{\lambda_{h b}}{2 \lambda+\lambda_{h b}} f_{\text {phh_busy }}^{*}(s) .
\end{aligned}
$$

4) The effective channel holding time of the new session and the handoff session have the same distribution if and only if the cell residence time is exponentially distributed. 


\section{Analytical Model of Conditional Distribution With Packet Session Channel HOLDING TIME}

We study the conditional distribution of new packet session holding time and handoff packet session holding time while the packet call completes in its current cell and the timer is timeout (the length of timer is $a$ ).

Let $f_{c n h}(t)$ and $f_{c h h}(t)$ denote the conditional density functions condition for the channel holding time of new packet session and the channel holding time of handoff packet session in busy model, respectively. The corresponding Laplace transforms of these density functions are $f_{c n h}^{*}(t)$ and $f_{c h h}^{*}(t)$, respectively; and with cumulative distribution functions $F_{c n h}(t)$ and $F_{c h h}(t)$. We first start from the channel holding time of handoff packet session condition on timer is timeout and $t_{j} \geq r_{j}+a$. we have

A. handoff session (in busy mode)

$$
\begin{aligned}
F_{c h h}(h) & =\operatorname{Pr}\left(t_{p h h_{-} \text {busy }} \leq h \mid r_{j}+a \leq t_{j}\right) \\
& =\frac{\int_{0}^{h-a} f_{p}(t) \int_{t+a}^{\infty} f(\tau) d \tau d t}{\operatorname{Pr}\left(r_{j}+a \leq t_{j}\right)} \\
& =\frac{\int_{0}^{h-a} f_{p}(t)\left[1-F_{d}(t+a)\right] d t}{\operatorname{Pr}\left(r_{j}+a \leq t_{j}\right)}
\end{aligned}
$$

Differentiating both sides, we obtain the conditional density function

$$
f_{c h h}(h)=\frac{f_{p}(h-a)\left[1-F_{d}(h)\right]}{\operatorname{Pr}\left(r_{j}+a \leq t_{j}\right)}
$$

We observe that

$$
\begin{aligned}
\operatorname{Pr}\left(r_{j}+a \leq t_{j}\right) & =\int_{t=a}^{\infty} \int_{0}^{t-a} f_{d}(t) f_{p}(\tau) d \tau d t \\
& =\int_{t=a}^{\infty} f_{d}(t)\left[1-e^{-\mu(t-a)}\right] d t \\
& =1-\int_{t=a}^{\infty} f_{d}(t) e^{-\mu(t-a)} d t \\
& =1-F_{d}(a)-e^{\mu a} f_{d}^{*}(\mu)
\end{aligned}
$$

Taking this into (28), we obtain

$$
f_{c h h}(h)=\frac{\left[1-F_{d}(h)\right] \mu e^{-\mu(h-a)}}{1-F_{d}(a)-e^{\mu a} f_{d}^{*}(\mu)}
$$

Hence,

$$
\begin{aligned}
f_{c h h}^{*}(s) & =\frac{\mu \int_{0}^{\infty} e^{-(s+\mu)(h-a)}\left[1-F_{d}(h)\right] d h}{1-F_{d}(a)-e^{\mu a} f_{d}^{*}(\mu)} \\
& =\frac{\mu e^{(s+\mu) a}}{s+\mu} \frac{1-f_{d}^{*}(s+\mu)}{1-F_{d}(a)-e^{\mu a} f_{d}^{*}(\mu)}
\end{aligned}
$$

\section{B. New Session}

In a similar fashion, we obtain the following result for the new session channel holding time:

$$
f_{c n h}(h)=\frac{\left[1-F_{r}(h)\right] \mu e^{-\mu(h-a)}}{1-F_{r}(a)-e^{\mu a} f_{r}^{*}(\mu)}
$$

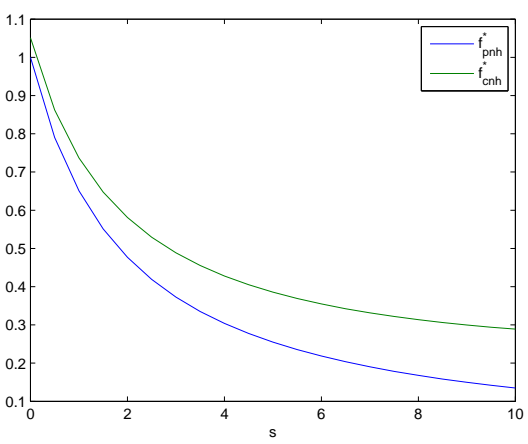

Figure 5. Compare of the Laplace transform function of unconditional and conditional probability density function of new session holding time.

$$
\begin{aligned}
f_{c n h}^{*}(s) & =\frac{\mu \int_{0}^{\infty} e^{-(s+\mu)(h-a)}\left[1-F_{r}(h)\right] d h}{1-F_{r}(a)-e^{\mu a} f_{r}^{*}(\mu)} \\
& =\frac{\mu e^{(s+\mu) a}}{s+\mu} \cdot \frac{1-f_{r}^{*}(s+\mu)}{1-F_{r}(a)-e^{\mu a} f_{r}^{*}(\mu)}
\end{aligned}
$$

The conditional channel holding time distribution $f_{c h}(t)$ is the (weighted) average of the conditional new session and handoff session channel holding time distribution.

$$
\begin{aligned}
f_{c h}^{*}(s) & =\frac{\mu e^{(s+\mu) a}}{s+\mu}\left(\frac{\lambda}{2 \lambda+\lambda_{h}} \cdot \frac{1-f_{r}^{*}(s+\mu)}{1-F_{r}(a)-e^{\mu a} f_{r}^{*}(\mu)}\right. \\
& \left.+\frac{\lambda_{h}}{2 \lambda+\lambda_{h}} \cdot \frac{1-f_{d}^{*}(s+\mu)}{1-F_{d}(a)-e^{\mu a} f_{d}^{*}(\mu)}\right)
\end{aligned}
$$

Let $T_{c n h}, T_{c h h}$, and $T_{c h}$ denote the expected conditional new session, handoff session, channel holding time, respectively, then we have

$$
\begin{aligned}
T_{c h h} & =\frac{-e^{\mu a}}{\mu}\left(\frac{1-\mu a-f^{*}(\mu)}{1-e^{\mu a} f^{*}(\mu)}+f^{*(1)}(\mu)\right) \\
T_{c n h} & =\frac{-e^{\mu a}}{\mu}\left(\frac{1-\mu a-f_{r}^{*}(\mu)}{1-e^{\mu a} f_{r}^{*}(\mu)}+f_{r}^{*(1)}(\mu)\right) \\
T_{c h}= & \frac{-e^{\mu a}}{\mu}\left\{\frac{1-\mu a-f_{r}^{*}(\mu)}{1-e^{\mu a} f_{r}^{*}(\mu)}+f_{r}^{*(1)}(\mu)\right) \\
+ & \left.\frac{\lambda_{h}}{2 \lambda+\lambda_{h}}\left(\frac{1-\mu a-f^{*}(\mu)}{1-e^{\mu a} f^{*}(\mu)}+f^{*(1)}(\mu)\right)\right\}
\end{aligned}
$$

When the residual life time $r_{1}$ of $t_{1}$ is exponentially distributed with parameter $\mu_{r}$, then its Laplace transform $f_{r}^{*}(s)=\mu_{r} /\left(s+\mu_{r}\right)$. From (31) we obtain

$$
\begin{aligned}
f_{c n h}^{*}(s) & =\frac{\mu e^{(s+\mu) a}}{s+\mu} \cdot \frac{1-f_{r}^{*}(s+\mu)}{1-F_{r}(a)-e^{\mu a} f_{r}^{*}(\mu)} \\
& =\frac{\mu e^{(s+\mu) a}}{s+\mu} \cdot \frac{\frac{s+\mu}{s+\mu+\mu_{r}}}{\frac{\mu \mu_{r}}{\mu+\mu_{r}}}=\frac{e^{(s+\mu) a}}{s+\mu+\mu_{r}} \cdot \frac{\mu+\mu_{r}}{\mu_{r}}
\end{aligned}
$$

In Fig. (5), we found that in the packet switch system, the new session holding time has different probability density distribution in unconditional and conditional distribution. 

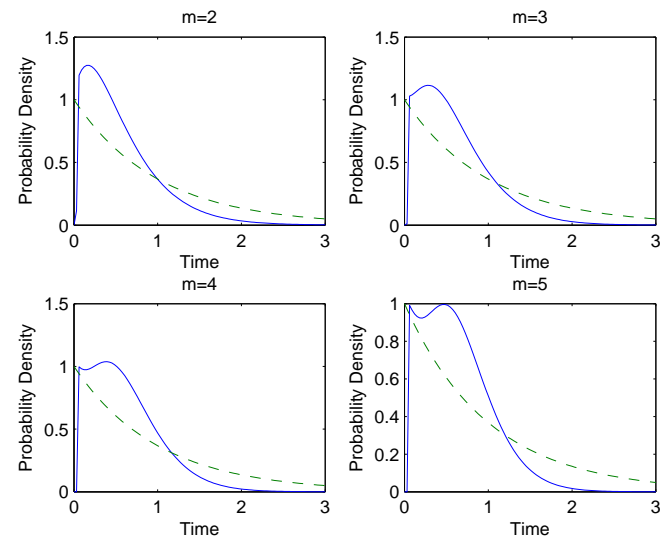

Figure 6. Probability density of handoff session holding time (solid line) and its exponential fitting (dashed line) when cell residence time is Erlang distributed with parameter $(\mu, \eta)$
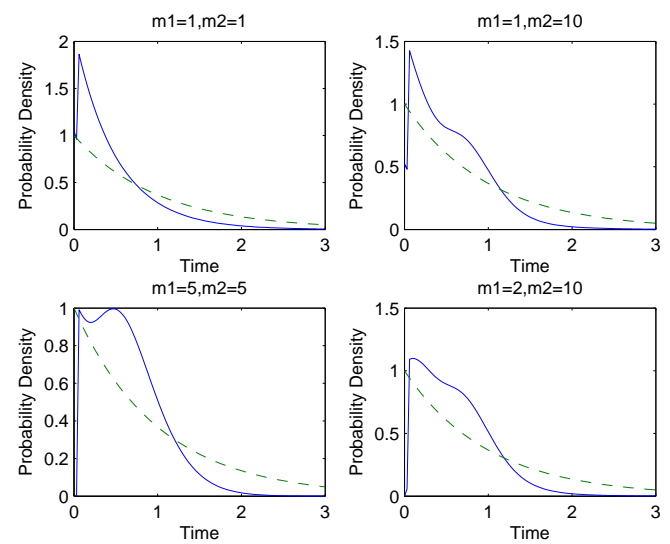

Figure 7. Probability density of handoff session holding time (solid line) and its exponential fitting (dashed line) when cell residence time is HyperErlang distributed with parameter $\left(m_{1}, m_{2}, \eta\right)$

\section{NumericAl RESUltS}

In 1996, Jedrrzycki and Leung showed that the distribution of circuit switch channel holding time is approximated with the log-normal distribution after removing the spikes of data [13]. Orlik and Rappaport derived the results for conditional distributions for the channel holding time when the cell residence time is SOHYP distributed [14], [15]. In 2007, Chen et al. observed that the mobile VoIP channel holding time distribution can be accurately approximated by a mix of two log-normal distributions [16]. To avoid complex computation with log-normal or SOHYP distribution in cell residence time, we use a more general distribution model for cell residence time.

In this section, we apply the Hyper-Erlang distribution model [2]-[4] to analyze the packet session channel holding time. Refer to [2]-[4], the Hyper-Erlang distribution has the following probability density function and Laplace trans- form:

$$
\begin{aligned}
f_{h e}(t) & =\sum_{i=1}^{M} \alpha_{i} \frac{\left(m_{i} \eta_{i}\right)^{m_{i}} t^{m_{i}-1}}{\left(m_{i}-1\right) !} e^{-m_{i} \eta_{i} t}, t \geq 0 \\
f_{h e}^{*}(s) & =\sum_{i=1}^{M} \alpha_{i}\left(\frac{m_{i} \eta_{i}}{s+m_{i} \eta_{i}}\right)^{m_{i}} .
\end{aligned}
$$

Furthermore, we need to investigate the relationship between $f_{r}^{*}(s)$ and $f_{d}^{*}(d)$. Because the residual life time $r_{1}$ can be viewed as the residual life of the cell residence time, from the Residual Life Theorem [10], [17] and [3], we obtain:

$$
f_{r}^{*}(s)=\frac{\eta\left[1-f_{d}^{*}(d)\right]}{s}
$$

where $\eta=\left[\sum_{i=1}^{M} \alpha_{i} / \eta_{i}\right]^{-1}$.

From [2]-[4], the density function and Laplace transform of the Hyper-Erlang distribution with one parameter are as follows:

$$
f(t)=\frac{\beta^{m} t^{m-1}}{(m-1) !} e^{-\beta t}, f^{*}(s)=\left(\frac{\beta}{s+\beta}\right)^{m}
$$

First, we use the Hyper-Erlang distribution model of one parameter for our numerical study. We take the Hyper-Erlang and exponential cell residence time into Theorem 2. We than can obtain the probability density of the handoff packet session holding time from our analytical results. In Fig. 6, we use one Erlang parameter and one exponential parameter in our Hyper-Erlang model. We vary the shape parameter $(m)$. It can be seen that the exponential model does not have good fit for the real handoff packet session holding time distribution when the shape parameter $(m)$ increases larger.

Second, we apply the cell residence time of Hyper-Erlang distributed with two parameters as follows:

$$
f^{*}(s)=\alpha_{1}\left(\frac{m_{1} \eta}{s+m_{1} \eta}\right)^{m_{1}}+\alpha_{2}\left(\frac{m_{2} \eta}{s+m_{2} \eta}\right)^{m_{2}}
$$

Fig. 7 shows the Hyper-Erlang distribution model with two parameters. This figure indicates more mismatches between the handoff packet session holding time distribution and its exponential fitting.

\section{SUMMARY}

In this paper, we propose a new packet session model with DRX idle mode to analyze the channel holding time for packet sessions in PS networks. We show that if cell residence time is exponentially distributed, packet session channel holding time is exponentially distributed. We also demonstrate that the cell residence time can capture user's mobility. Besides, the Hyper-Erlang distribution models can be used to characterize user's mobility when a PS network has exponential packet session holding time, Poisson new session arrival, and Hyper-Erlang cell residence time. 


\section{ACKNOWLEDGMENTS}

This work was supported in part by the Ministry of Science and Technology of Taiwan under grant numbers NSC 101-2628-E-009-015-MY3 and MOST 103-2221-E009-110-MY3.

\section{REFERENCES}

[1] Z. Ying, "User Mobility from the View of Cellular Data Networks," in Proceedings of the 33rd Annual IEEE Conference on Computer Communications, 2014, pp. 1348-1356.

[2] Y. Fang, I. Chlamtac, and Y. B. Lin, "Channel Occupancy Times and Handoff Rate for Mobile Computing and PCS Networks," IEEE Transactions on Computers, vol. 47, no. 6, pp. $679-692,1998$.

[3] Y. Fang and I. Chlamtac, "Teletraffic analysis and mobility modeling of PCS networks," IEEE Transactions on Communications, vol. 47, no. 7, pp. 1062 - 1072, 1999.

[4] Y. Fang, "Hyper-Erlang distribution model and its application in wireless mobille networks," Wireless Networks - Special issue: Design and modeling in mobile and wireless systsems, vol. 7, no. 3, pp. $211-219,2001$.

[5] C. S. Bontu and E. Illidge, "DRX Mechanism for Power Saving in LTE," IEEE Communications Magazine, vol. 47, no. 6, pp. 48-55, 2009.

[6] A. L. E. Corral-Ruizl, F. A. Cruz-Pérez, and G. HernandezValdez, "Channel holding time in mobile cellular networks with generalized Coxian distributed cell dwell time," in IEEE International Symposium on Personal, Indoor and Mobile Radio Communications, PIMRC, 2010, pp. 2348 - 2353.

[7] A. L. E. Corral-Ruiz, A. Rico-Páez, F. A. Cruz-Pérez, and G. Hernández-Valdez, "On the Functional Relationship between Channel Holding Time and Cell Dwell Time in Mobile Cellular Networks," in GLOBECOM - IEEE Global Telecommunications Conference (2010), 2010, pp. 1 - 6.

[8] A. L. E. Corral-Ruiz, F. A. Cruz-Perez, and G. HernandezValdez, "Cell Dwell Time and Channel Holding Time Relationship in Mobile Cellular Networks," in Cell Dwell Time and Channel Holding Time Relationship in Mobile Cellular Networks, Wireless Communications and Networks - Recent Advances, Dr. Ali Eksim (Ed.), 2012.

[9] M. M. Zonoozi and P. Dassanayake, "User Mobility Modeling and Characterization of Mobility Patterns," Selected Areas in Communications, IEEE Journal on, vol. 15, no. 7, pp. 12391252, 1997.

[10] K. L., Queueing Systems: Theory. New York: Wiley, 1979.

[11] E. D. Re, R. Fantacci, and G. Giambene, "Efficient dynamic channel allocation techniques with handover queueing for mobile satellite networks," IEEE Journal on Selected Areas in Communications, vol. 13, no. 2, pp. 704-712, 1994.

[12] S. Sae-ung and S. Lertprapai, "A Comparision of Scale Parameter Estimators in the 2-Parameter Exponential Distribution Based on Mutiple Criteria Decsion Making," Thai Journal of Mathematics, vol. 8, no. 4, pp. 73-82, 2010.
[13] C. Jedrzycki and V. Leung, "Probability distributions of channel holding time in cellular telephony systems," Proceedings of Vehicular Technology Conference - VTC, pp. 247-251, 1996.

[14] P. V. Orlik and S. S. Rappaport, "A model for teletrafc performance and channel holding time characterization in wireless cellular communication with general session and dwell time distributions," IEEE Journal on Selected Areas in Communications, vol. 16, pp. 788-803, 1998.

[15] _ , "A model for teletrafc performance and channel holding time characterization in wireless cellular communication," in Universal Personal Commun., 1997, pp. 671-675.

[16] W. E. Chen, H. N. Hung, and Y. B. Lin, "Modeling VoIP Call Holding Times for Telecommunications," Network, IEEE, vol. 21 , no. 6 , pp. $22-28,2007$.

[17] J. Medhi, Stochastic Models in Queueing Theory. New York: Academic Press, 1991

[18] J. L. Schiff, The Laplace transform - Theory and Applications. Springer, 1999.

\section{APPENDIX}

Simple poles [18]: Suppose that we have Laplace transform $F(S)=\mathcal{L}(f(t))$ for

$F(s)=\frac{P(s)}{Q(s)}=\frac{P(s)}{\left(s-\alpha_{1}\right)\left(s-\alpha_{2}\right) \cdots\left(s-\alpha_{n}\right)}, \quad \alpha_{i} \neq \alpha_{j}$,

where $P(s)$ is a polynomial of degree less than $n$. In the terminology of complex variables, the $\alpha_{i} s$ are known as simple poles of $F(s)$. A partial fraction decomposition is:

$$
F(s)=\frac{A_{1}}{\left(s-\alpha_{1}\right)}+\frac{A_{2}}{\left(s-\alpha_{2}\right)}+\cdots+\frac{A_{n}}{\left(s-\alpha_{n}\right)} .
$$

Multiplying both sides of (41) by $s-\alpha_{i}$ and letting $s \rightarrow \alpha_{i}$ yield:

$$
A_{i}=\lim _{s \rightarrow \alpha_{i}}\left(s-\alpha_{i}\right) F(s) .
$$

Therefore,

$$
f(t)=\mathcal{L}^{-1}(F(s))=\sum_{i=1}^{n} \mathcal{L}^{-1}\left(\frac{A_{i}}{s-\alpha_{i}}\right)=\sum_{i=1}^{n} A_{i} e^{\alpha_{i} t} .
$$

Putting in (42) for $A_{i}$ gives a quick method to find the inverse:

$$
f(t)=\mathcal{L}^{-1}(F(s))=\sum_{i=1}^{n} \lim _{s \rightarrow \alpha_{i}}\left(s-\alpha_{i}\right) F(s) e^{\alpha_{i} t} .
$$

Applying to (21) to find:

$\mathcal{L}^{-1}\left(\frac{\mu_{r} s}{(s+\mu)\left(s+\mu+\mu_{r}\right)}\right)=\mathcal{L}^{-1}\left(\frac{\mu_{r} s}{(s-(-\mu))\left(s-\left(\mu-\mu_{r}\right)\right)}\right)$.

Thus, from (43) we can obtain:

$f(t)=\mathcal{L}^{-1}(F(s))$

$=\lim _{s \rightarrow-\mu}(s+\mu) F(s) e^{-\mu t}+\lim _{s \rightarrow-\mu-\mu_{r}}\left(s+\mu+\mu_{r}\right) F(s) e^{-\left(\mu+\mu_{r}\right) t}$

$=\frac{-\mu \cdot \mu_{r}}{-\mu+\mu+\mu_{r}} \cdot e^{-\mu t}+\frac{\mu_{r} \cdot\left(-\mu-\mu_{r}\right)}{\left(-\mu-\mu_{r}\right)+\mu} \cdot e^{-\left(\mu+\mu_{r}\right) t}$. 